\title{
Strong Unitary and Overlap Uncertainty Relations: Theory and Experiment
}

\author{
Kok-Wei Bong, ${ }^{1}$ Nora Tischler, ${ }^{1}$ Raj B. Patel, ${ }^{1}$ Sabine Wollmann, ${ }^{1,2}$ Geoff J. Pryde, ${ }^{1, *}$ and Michael J. W. Hall ${ }^{1,3}$ \\ ${ }^{1}$ Centre for Quantum Computation and Communication Technology (Australian Research Council), \\ Centre for Quantum Dynamics, Griffith University, Brisbane QLD 4111, Australia \\ ${ }^{2}$ Quantum Engineering Technology Labs, H. H. Wills Physics Laboratory and Department of Electrical and Electronic Engineering, \\ University of Bristol, Bristol BS8 1FD, United Kingdom \\ ${ }^{3}$ Department of Theoretical Physics, Research School of Physics and Engineering, Australian National University, \\ Canberra ACT 0200, Australia
}

(Received 21 November 2017; revised manuscript received 18 December 2017; published 5 June 2018)

We derive and experimentally investigate a strong uncertainty relation valid for any $n$ unitary operators, which implies the standard uncertainty relation and others as special cases, and which can be written in terms of geometric phases. It is saturated by every pure state of any $n$-dimensional quantum system, generates a tight overlap uncertainty relation for the transition probabilities of any $n+1$ pure states, and gives an upper bound for the out-of-time-order correlation function. We test these uncertainty relations experimentally for photonic polarization qubits, including the minimum uncertainty states of the overlap uncertainty relation, via interferometric measurements of generalized geometric phases.

DOI: 10.1103/PhysRevLett.120.230402

Introduction.-Uncertainty relations are one of the most important foundations of physics, defining the limits on what is possible in a quantum world. Their implications range from bounds in quantum metrology [1,2], through the security of quantum cryptographic schemes $[3,4]$, and to the measurement and control of deeply quantum systems [5]. We present a very powerful, yet simple, uncertainty relation for the reversible transformations of a quantum system (represented by unitary operators), which unifies, generalizes, and significantly strengthens previous results. For example, our unitary uncertainty relation for $n$ operators (i) is saturated by every pure state of an $n$-dimensional Hilbert space (and by all pure qubit states), (ii) is stronger than, and can be used to derive, the standard Heisenberg and Robertson-Schrödinger uncertainty relations $[6,7]$ and various others in the literature [8-13], (iii) leads to an upper bound for the out-of-time-order correlator-of strong interest in quantum thermalization, chaos, and information scrambling, for both many-body and black-hole physics [14-19], and (iv) generates a strong inequality for the transition probabilities connecting any $n+1$ pure states. Our relation can therefore be viewed as an "ur"-uncertainty relation, which unifies a number of seemingly disparate quantum concepts. We experimentally investigate this uncertainty relation, and its implications for transition probabilities, via robust interferometric measurements of generalized geometric phases [20-24] on polarization qubits (extendable to any $n$ unitaries).

Unitary uncertainty relation.-For $n$ unitary operators $U_{1}, U_{2}, \ldots, U_{n}$ and quantum state $\rho$, define $U_{0}=I$ and $v^{(j)}=U_{j} \rho^{1 / 2}$. For any given set of $n+1$ vectors $\left\{v^{(j)}\right\}$ with inner product $(\cdot, \cdot)$, the corresponding Gram matrix $G$, with coefficients $G_{j k}=\left(v^{(j)}, v^{(k)}\right)$, is positive semidefinite [25]. Hence, choosing the inner product $(A, B)=\operatorname{Tr}\left[A^{\dagger} B\right]$ on the vector space of linear operators, one has the unitary uncertainty relation (UUR)

$$
\operatorname{det} G \geq 0, \quad G_{j k}:=\operatorname{Tr}\left[\rho U_{j}^{\dagger} U_{k}\right]=\left\langle U_{j}^{\dagger} U_{k}\right\rangle
$$

(and, more generally, the stronger relation $G \geq 0$ ). We note that a similar method was used by Robertson to obtain an uncertainty relation for $n$ Hermitian operators and a pure state [26]. For $n=2$, the UUR reduces to

$$
\operatorname{Var} U \operatorname{Var} V \geq\left|\left\langle U^{\dagger} V\right\rangle-\left\langle U^{\dagger}\right\rangle\langle V\rangle\right|^{2}
$$

for two unitary operators $U$ and $V$, recently obtained elsewhere by less simple means $[12,13]$, where the variance of unitary operator $U$ is defined by $\operatorname{Var} U:=1-|\langle U\rangle|^{2}$. As well as a direct measure of uncertainty, vanishing only for eigenstates of $U$ [27], $\operatorname{Var} U$ quantifies the disturbance of pure states by $U$ : it reaches its minimum value of 0 for a nondisturbing rephasing of the state, $U|\psi\rangle=\exp (i \theta)|\phi\rangle$, and its maximum value of 1 for the maximally disturbing case that $U$ transforms $|\psi\rangle$ to an orthogonal state.

The $n=3$ case is discussed in the Supplemental Material [28]. It is further shown there that expanding $U=e^{i \epsilon A}, V=e^{i \epsilon B}$ in $\epsilon$ in Eq. (2) yields the standard Robertson-Schrödinger uncertainty relation $[6,7]$

$$
\operatorname{Var} A \operatorname{Var} B \geq \frac{1}{4}|\langle[A, B]\rangle|^{2}+\operatorname{Cov}(A, B)^{2},
$$

for two observables represented by Hermitian operators $A$ and $B$ (with the quantum covariance defined by 
$\left.\operatorname{Cov}(A, B):=\frac{1}{2}\langle A B+B A\rangle-\langle A\rangle\langle B\rangle\right)$, and that several recent uncertainty relations [8-11] can also be obtained from the UUR in Eq. (1).

To determine the states that saturate the UUR, i.e., its minimum uncertainty states, note that the determinant of a Gram matrix vanishes if and only if the vectors $v^{(j)}$ are linearly dependent [25]. For a pure state $\rho=|\psi\rangle\langle\psi|$, this is equivalent to linear dependence of $|\psi\rangle, U_{1}|\psi\rangle, \ldots, U_{n}|\psi\rangle$, which is always satisfied for the case of a Hilbert space with dimension $d \leq n$. Hence, every pure state is a minimum uncertainty state for this case, emphasizing the strength of the UUR. In particular, Eq. (1), and hence Eqs. (2) and (3), are saturated by all qubit pure states. Conversely, a mixed qubit state is a minimum uncertainty state of Eq. (2) if and only if $[U, V]=0$, i.e., if and only if $U$ and $V$ correspond to rotations about the same axis of the Bloch sphere (see Supplemental Material [28]).

The UUR is invariant under $U_{j} \rightarrow e^{i \phi_{j}} U_{j}$ [even though $G_{j k}$ in Eq. (1) is not], i.e., under physically equivalent unitaries [28]. Indeed, for a pure state $|\psi\rangle$, Eq. (1) can be rewritten in terms of the Bargmann projective invariants $B_{j_{1} \ldots j_{r}}:=\operatorname{Tr}\left[\left|\psi_{j_{1}}\right\rangle\left\langle\psi_{j_{1}}|\ldots| \psi_{j_{r}}\right\rangle\left\langle\psi_{j_{r}}\right|\right]$, invariant under rephasings $\left|\psi_{j_{r}}\right\rangle \rightarrow e^{i \phi_{j_{r}}}\left|\psi_{j_{r}}\right\rangle$, where $\left|\psi_{j+1}\right\rangle:=U_{j}|\psi\rangle$ $[21,28]$ (these invariants are closely related to geometric phases [24]). For example, Eq. (2) becomes

$$
\cos \Phi \geq \frac{T_{12}+T_{13}+T_{23}-1}{2 \sqrt{T_{12} T_{13} T_{23}}},
$$

where $T_{j k}=\left|\left\langle\psi_{j} \mid \psi_{k}\right\rangle\right|^{2}=B_{j k}$ is the transition probability between $\left|\psi_{j}\right\rangle$ and $\left|\psi_{k}\right\rangle$, and $\Phi$ is the phase of the complex number $B_{123}$. The saturation of this inequality for all pure qubit states corresponds to an identity in spherical trigonometry $[28,29]$. For general mixed states, the UUR can be tested via the measurement of suitably generalized Bargmann invariants, as reported below. In particular, Eq. (2) is equivalent to

$$
\cos \Phi \geq \frac{|\langle U\rangle|^{2}+|\langle V\rangle|^{2}+\left|\left\langle U^{\dagger} V\right\rangle\right|^{2}-1}{2\left|\langle U\rangle\left\langle U^{\dagger} V\right\rangle\left\langle V^{\dagger}\right\rangle\right|},
$$

generalizing Eq. (4), where $\Phi$ is the phase of the generalized Bargmann invariant $\langle U\rangle\left\langle U^{\dagger} V\right\rangle\left\langle V^{\dagger}\right\rangle$ [28].

Overlap uncertainty relation.-Overlap uncertainty relations reflect the nonclassical property that even pure quantum states typically overlap, important for quantum state discrimination and quantum metrology $[1,2,30,31]$ and in SWAP-tests [32] for quantum communication [33]. For example, the overlap between two phase-shifted optical modes, $|\psi\rangle$ and $e^{-i N \chi}|\psi\rangle$, is $T_{\chi}=\left|\left\langle\psi\left|e^{-i N \chi}\right| \psi\right\rangle\right|^{2}=$ $1-\chi^{2}(\Delta N)^{2}+O\left(\chi^{4}\right)$, implying that a small overlap, $T_{\chi} \ll 1$, as required to resolve a small phase shift $\chi$, requires a large photon number uncertainty $\Delta N \gtrsim 1 / \chi$. Our unitary uncertainty relation unifies quantum limits on state preparation and overlap, by generating a tight overlap uncertainty relation for any given set of $n+1$ pure states.

For example, noting that $\cos \Phi \leq 1$, Eq. (4) immediately yields the overlap uncertainty relation (OUR)

$$
T_{12}+T_{13}+T_{23}-2 \sqrt{T_{12} T_{13} T_{23}} \leq 1
$$

for the transition probabilities connecting any three pure states. This relation is tight, being saturated if and only if the states lie on a geodesic in Hilbert space, and for qubits, this corresponds to their Bloch vectors lying on a great circle [28]. It is also a very strong constraintstronger, e.g., than the overlap uncertainty relation $\sqrt{1-T_{12}} \leq \sqrt{1-T_{13}}+\sqrt{1-T_{23}}$, for the transition probabilities of any three pure states $\left|\psi_{1}\right\rangle,\left|\psi_{2}\right\rangle,\left|\psi_{3}\right\rangle$, corresponding to the triangle inequality for trace distance [28,31]. For states $|\psi\rangle, U|\psi\rangle, V|\psi\rangle$, with a fixed $U$ and $V$, saturation of the OUR determines the corresponding minimum uncertainty states $\{|\psi\rangle\}$, as investigated experimentally below. More generally, the UUR (1) generates an overlap uncertainty relation for $n+1$ states, explored further in the Supplemental Material [28].

Experimental setup.-Our experiment uses polarization states of single photons and a displaced Sagnac interferometer with controllable unitary transformations, $U$ in the transmitted arm and $V$ in the reflected arm (see Fig. 1). We can determine the value of $\left\langle U^{\dagger} V\right\rangle$ for an input state $\rho$ by first noting that the average output photon number is given by $\langle N\rangle_{\chi}=\frac{1}{2}\left[1+\operatorname{Re}\left\{e^{-i \chi}\left\langle U^{\dagger} V\right\rangle\right\}\right]$, where $\chi$ is the phase difference between the two arms. Hence, we can obtain an interference pattern by varying $\chi$, with associated visibility

$$
\mathcal{V}(U, V):=\frac{\langle N\rangle_{\max }-\langle N\rangle_{\min }}{\langle N\rangle_{\max }+\langle N\rangle_{\min }}=\left|\left\langle U^{\dagger} V\right\rangle\right|
$$

The values of $|\langle U\rangle|$ and $|\langle V\rangle|$ are similarly determined from the corresponding visibilities $\mathcal{V}(U, I), \mathcal{V}(I, V)$, where $I$ denotes the identity transformation. Further, the phase of $\left\langle U^{\dagger} V\right\rangle$ corresponds to the value of the phase difference $\chi$ that gives a maximum average output photon number (for a pure input state $|\psi\rangle$, this value is the Pancharatnam phase between $U|\psi\rangle$ and $V|\psi\rangle[20,34])$. If $\chi(U, V)$ denotes the location of the interference maximum relative to some fixed phase reference value $\chi_{0}$, it follows that the phase of $\left\langle U^{\dagger} V\right\rangle$ is given by

$$
\arg \left\langle U^{\dagger} V\right\rangle=\chi(U, V)-\chi(I, I) .
$$

Thus, our setup allows us to extract $\left\langle U^{\dagger} V\right\rangle$ from the interference pattern via Eqs. (7) and (8). More generally, this setup allows the Gram matrix coefficients $G_{j k}$ in Eq. (1) to be experimentally determined for any set of unitary transformations $U_{0}=I, U_{1}, \ldots U_{n}$ and polarization state $\rho$, and hence, the testing of the UUR for any $n$. We note that, in comparison, a recent qutrit experiment testing a special 


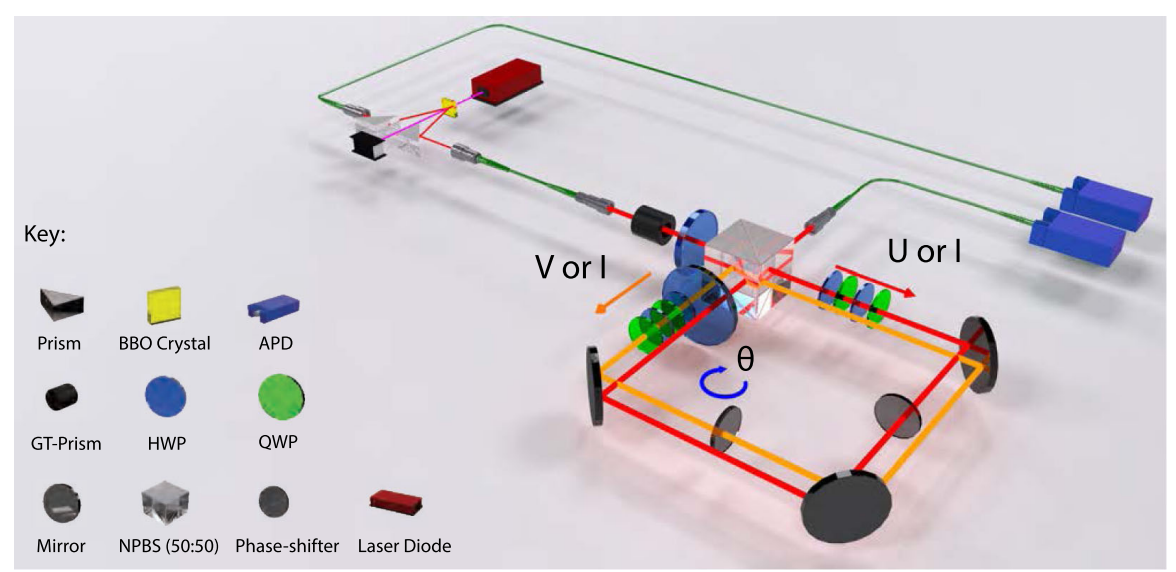

FIG. 1. Experimental setup for testing the unitary and overlap uncertainty relations. Pairs of single photons are generated via SPDC using a type-I BBO crystal. The signal photon is prepared in an arbitrary linear polarization state using a Glan-Taylor (GT) prism followed by a half-wave plate (HWP). After entering a displaced Sagnac interferometer at a 50:50 nonpolarizing beam splitter (NPBS), the photon traverses the interferometer in a superposition of the transmitted (red) and reflected (orange) paths. Unitary operators $U, V$, and $I$ are implemented using HWPs and quarter-wave plates (QWP). An additional HWP compensates for the birefringent phase upon reflection at the NPBS. A glass element in one path is tilted to act as a phase shifter, while a fixed element in the other path keeps the path-length difference to within the coherence length. Two avalanche photodiodes (APDs) detect the signal and idler (heralding) photons.

case of the $n=2$ UUR requires preparation of a strictly pure state $|\psi\rangle$, prior knowledge of the unitary operators (to implement both $V$ and $V^{\dagger}$ ), and tomographic reconstruction of $|\psi\rangle, U|\psi\rangle$, and $V|\psi\rangle$ [35].

As shown in Fig. 1, the main component of our setup is the displaced Sagnac interferometer, which is used to measure visibilities and phases as above. For the singlephoton source, we use a $410 \mathrm{~nm}$ continuous-wave diode laser to pump an optically nonlinear beta Barium Borate (BBO) crystal. The degenerate photon pairs generated by the noncollinear type-I spontaneous parametric down conversion (SPDC) are collected into optical fibers. The idler photon heralds the presence of a signal photon. A halfwave plate (HWP) allows for a range of polarization qubit states to be encoded on the signal photon, which is then sent into the interferometer. Each unitary operator, $U$ and $V$, is implemented by a combination of HWPs and quarter-wave plates (QWP), arranged in a group of four: HWP/QWP/HWP/QWP (Fig. 1), with the QWPs set to $45^{\circ}$, and the HWPs at variable angles $\alpha$ and $\beta$. The condition $\alpha=90^{\circ}$ and $\beta=0^{\circ}$ corresponds to implementing the identity operation. To realize an adjustable phase shift, a glass element is mounted on a motorized tilt controller and inserted in one arm of the interferometer. A fixed glass element is positioned in the other arm in order to keep the path-length differences to within the coherence length. Finally, the photons are detected by silicon avalanche photodiodes.

Results.-The interference fringes in Fig. 2 are obtained by measuring the photon counts at the output of the interferometer. The wave plates in one arm of the interferometer are rotated to produce either the identity operation $I$ or an operation $U\left(\alpha_{U}, \beta_{U}\right)$ specified by $\alpha_{U}$ and $\beta_{U}$.
Similarly, $V\left(\alpha_{V}, \beta_{V}\right)$ or $I$ can be implemented in the other arm. The left- and right-hand sides of the UUR in Eq. (5) are calculated by measuring four interference fringes, as shown in Fig. 2. We extract the phase and the visibility of the interference fringes by fitting the data to $A_{1}+A_{2} \cos ^{2}\left[\frac{1}{2}\left(\theta-\theta_{0}\right)\right]$, where $\theta=\chi-\chi_{0}$ is the controlled phase shift implemented by the tilted glass element. The visibility is then given by $\mathcal{V}(U, V)=A_{2} /\left(2 A_{1}+A_{2}\right)$, and $\chi(U, V)$ by $\theta_{0}$. The phase $\Phi$ of the generalized Bargmann invariant $\langle U\rangle\left\langle U^{\dagger} V\right\rangle\left\langle V^{\dagger}\right\rangle$ follows via Eq. (8) as

$$
\Phi=\chi(U, V)-\chi(U, I)-\chi(I, V)+\chi(I, I)
$$

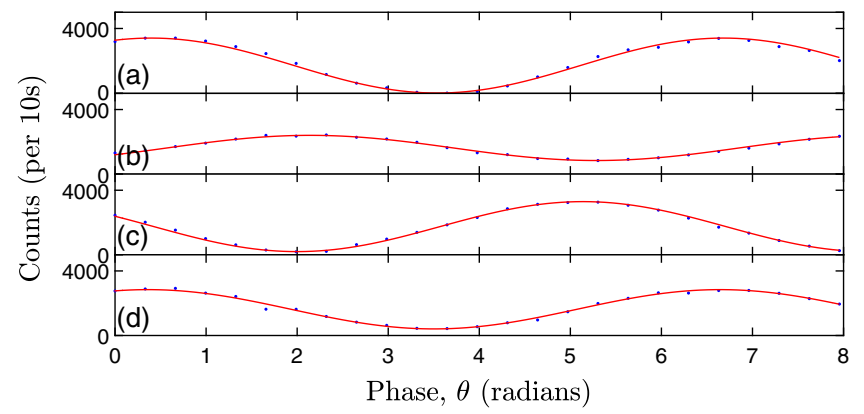

FIG. 2. Interferograms as recorded by measuring coincidence counts as a function of the applied phase shift. Unitaries $U, V$, or $I$ are applied to each arm of the Sagnac interferometer as follows a) Transmitted: $I$, Reflected: $I$. b) Transmitted: $U$, Reflected: $I$. c) Transmitted: $I$, Reflected: $V$. d) Transmitted: $U$, Reflected: $V$. The $\left|\left\langle U^{\dagger} V\right\rangle\right|$ terms or transition probabilities in the uncertainty relations can be calculated from the visibilities of the curves via Eq. (7), and the Bargmann phase via the phase terms in Eq. (9), each of which is determined from the phase of a fringe pattern. 


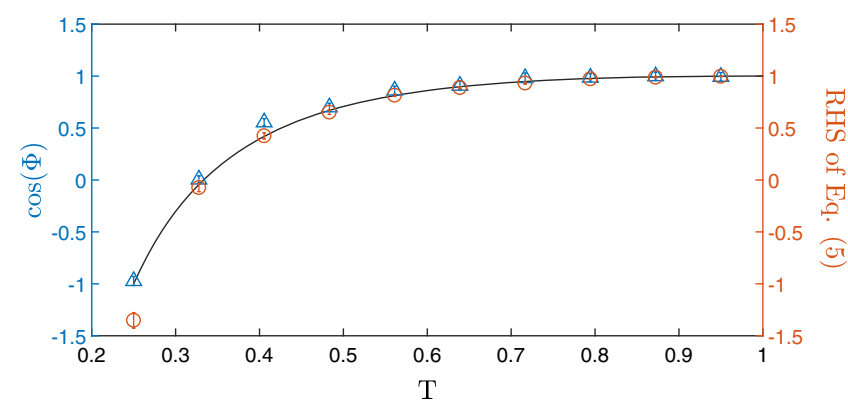

FIG. 3. Verification of the UUR in the form of Eq. (5), and its saturation by a pure qubit state. Using a fixed input state, $U$ and $V$ are varied pairwise over a range as described in the text. At each step, parametrized by the modsquared expectation value $T$ of the operators, the left-hand (blue triangles) and right-hand (red circles) sides of the UUR are determined from experimental measurements. The black line represents the theoretical prediction for saturation of Eq. (5). The errors are calculated from the standard errors of the fit parameters.

Verification of the UUR in Eq. (5) can be seen in Fig. 3. Here, we fix the input state to be horizontally polarized, $|H\rangle$. We vary $U$ and $V$ pairwise in steps such that the full range of $\cos \Phi$ is sampled and, at each setting, the tips of the Bloch vectors for $\{|H\rangle, U|H\rangle, V|H\rangle\}$ form an equilateral spherical triangle. This corresponds to $T_{12}=T_{23}=$ $T_{13}$ in Eq. (4). Saturation of Eq. (4) by pure qubit states corresponds to the area of the triangle being equal to $\Phi / 2$ [22-24,28]). In practice, there are small experimental imperfections. Although the states have high purity, they are not completely mixture free, and so Eq. (5) replaces Eq. (4) as the relevant UUR; also the nominal equilateral configuration is not exact. Nevertheless, since $|\langle U\rangle|^{2} \approx$ $|\langle V\rangle|^{2} \approx\left|\left\langle U^{\dagger} V\right\rangle\right|^{2}$, we write the average of these quantities as $T$, which forms the $x$ axis in Fig. 3. In the ideal pure state case for this configuration, $T=T_{i j}(\forall i \neq j)$ and $T_{12}=|\langle U\rangle|^{2}$, etc.

The experimental procedure to test the OUR in Eq. (6) uses a set of linearly polarized input states of high purity, and a fixed $U\left(\alpha_{U}=36^{\circ}, \beta_{U}=0^{\circ}\right)$ and $V\left(\alpha_{V}=0^{\circ}\right.$, $\left.\beta_{V}=36^{\circ}\right)$. The transition probabilities in Eq. (6) may be determined from the measured visibilities in Eq. (7), via $T_{j k}=\left|\left\langle U_{j}^{\dagger} U_{k}\right\rangle\right|^{2}=\mathcal{V}\left(U_{j}, U_{k}\right)^{2}$ for a pure input state. The results are shown in Fig. 4. The minimum uncertainty states correspond to the upper bound of unity in Eq. (6). We note that one of the sources of error in our experiments is the imperfect calibration and retardation of the wave plates, which leads to the implemented unitary operations deviating slightly from the expected settings.

Out-of-time-order correlators.-The UUR may also be used to obtain a bound for the out-of-time-order correlator (OTOC), $F=\left\langle W_{t}^{\dagger} V^{\dagger} W_{t} V\right\rangle$, for a fixed unitary $V$ and a time-dependent unitary $W_{t}$. The OTOC determines the disturbance caused by $V$ on a later measurement of $W$ and, as noted in the Introduction, is of interest in quantum thermalization, chaos, and information scrambling, both in

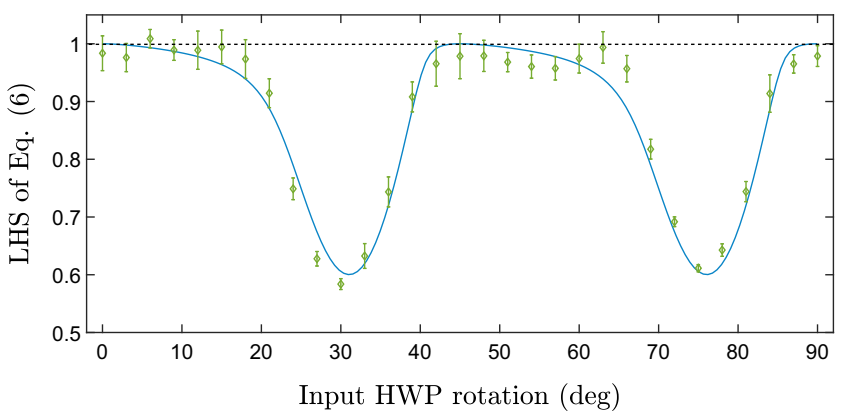

FIG. 4. Experimental test of the OUR in Eq. (6). The unitary operators in both arms of the interferometer remain fixed, and we input a family of states lying in the linear polarization plane. The green data points represent the left-hand side of Eq. (6). The blue solid line represents the theoretical curve of the left-hand side of Eq. (6). Minimum uncertainty states correspond to a value of unity, which is marked by the black dashed line. The error bars are calculated from the standard errors of the fit parameters.

many-body and black-hole physics [14-16]. It has only very recently been experimentally measured for some systems [17-19].

In particular, the UUR in Eq. (2) implies $\left(1-u^{2}\right)$ $\left(1-v^{2}\right) \geq\left(\left|\left\langle U^{\dagger} V\right\rangle\right|-u v\right)^{2}$, with $u=|\langle U\rangle|, \quad v=|\langle V\rangle|$, yielding

$$
\left|\left\langle U^{\dagger} V\right\rangle\right| \leq u v+\sqrt{\left(1-u^{2}\right)\left(1-v^{2}\right)}=\cos \left(\theta_{U}-\theta_{V}\right),
$$

with $\theta_{U}:=\cos ^{-1}|\langle U\rangle|$. Replacing $U$ by $W_{t} V$ and $V$ by $V W_{t}$ then gives the upper bound

$$
|F|=\left|\left\langle W_{t}^{\dagger} V^{\dagger} W_{t} V\right\rangle\right| \leq \cos \left(\theta_{V W_{t}}-\theta_{W_{t} V}\right)
$$

for the modulus of the OTOC, which shows that $|F|$ is a direct signature of the noncommutativity of $V$ and $W_{t}$. Indeed, using $\operatorname{Re}\{F\} \leq|F|$ yields the lower bound

$$
\left\langle\left|\left[V, W_{t}\right]\right|^{2}\right\rangle=2(1-\operatorname{Re}\{F\}) \geq 4 \sin ^{2}\left(\frac{\theta_{V W_{t}}-\theta_{W_{t} V}}{2}\right),
$$

where $\left\langle|A|^{2}\right\rangle$ denotes $\left\langle A^{\dagger} A\right\rangle$. For polarization qubits, we note that the values of $\theta_{V W_{t}}$ and $\theta_{W_{t} V}$ could be obtained from interferometer visibilities corresponding to $\left|\left\langle V W_{t}\right\rangle\right|$ and $\left|\left\langle W_{t} V\right\rangle\right|$, via Eq. (7), with a time-dependent unitary in one arm.

Conclusion.-We have presented a strong and very general unitary uncertainty relation (UUR), which implies the Robertson-Schrödinger relation and generates a tight state overlap uncertainty relation. We tested these experimentally using polarization qubit states in an interferometric configuration. This allowed for measurements that led directly to the quantities in the relation, and directly 
revealed the role of geometric phase in the UUR. We note that the UUR does not assume or require pure states, making it a general and powerful tool for real-world quantum systems.

We expect that the strength of the general UUR in Eq. (1) will lead to further results that enhance and unify quantum uncertainty relations. For example, noting that spin- $1 / 2$ observables are both Hermitian and unitary, the UUR in Eq. (2) leads directly to, and hence encompasses, a tight state-independent qubit uncertainty relation obtained recently in Refs. $[9,10]$, and it leads to a generalization of the uncertainty relation for characteristic functions in Ref. [11] (see Supplemental Material [28]). It would also be of interest in future work to investigate possible connections of the UUR with entropic, measurement-disturbance, and joint-measurement uncertainty relations, to test the UUR and OUR for higher values of $n$, and to implement similar tests of the OTOC bounds in Eqs. (11) and (12) above.

We thank J. Kaniewski for pointing out the connection between the UUR and the uncertainty relation in Ref. [8]. This work was supported by the ARC Centres of Excellence CE110001027 and CE170100012. S.W. acknowledges financial support through an Australian Government Research Training Program Scholarship.

*g.pryde@griffith.edu.au

[1] S. L. Braunstein and C. M. Caves, Phys. Rev. Lett. 72, 3439 (1994).

[2] S. L. Braunstein, C. M. Caves, and G. J. Milburn, Ann. Phys. (N.Y.) 247, 135 (1996).

[3] M. Berta, M. Christandl, R. Colbeck, J. M. Renes, and R. Renner, Nat. Phys. 6, 659 (2010).

[4] M. Tomamichel and R. Renner, Phys. Rev. Lett. 106, 110506 (2011).

[5] E.g., E. E. Wollman, C. U. Lei, A. J. Weinstein, J. Suh, A. Kronwald, F. Marquardt, A. A. Clerk, and K. C. Schwab, Science 349, 952 (2015).

[6] H. P. Robertson, Phys. Rev. 35, 667A (1930).

[7] E. Schrödinger, Ber. Kgl. Akad. Wiss. Berlin 24, 296 (1930).

[8] J. Kaniewski, M. Tomamichel, and S. Wehner, Phys. Rev. A 90, 012332 (2014).

[9] J.-L. Li and C.-F. Qiao, Sci. Rep. 5, 12708 (2015).
[10] A. A. Abbott, P.-L. Alzieu, M. J. W. Hall, and C. Branciard, Mathematics 4, 8 (2016).

[11] L. Rudnicki, D. S. Tasca, and S. P. Walborn, Phys. Rev. A 93, 022109 (2016).

[12] M. J. W. Hall, A. K. Pati, and J. Wu, Phys. Rev. A 93, 052118 (2016)

[13] S. Bagchi and A. K. Pati, Phys. Rev. A 94, 042104 (2016).

[14] P. Hosur, X.-L. Qi, D. A. Roberts, and B. Yoshida, J. High Energy Phys. 02 (2016) 4.

[15] J. Maldacena, S. H. Shenker, and D. Stanford, J. High Energy Phys. 08 (2016) 106.

[16] B. Swingle, G. Bentsen, M. Schleier-Smith, and P. Hayden, Phys. Rev. A 94, 040302 (2016).

[17] J. Li, R. Fan, H. Wang, B. Ye, B. Zeng, H. Zhai, X. Peng, and J. Du, Phys. Rev. X 7, 031011 (2017).

[18] M. Gärttner, J. G. Bohnet, A. Safavi-Naini, M. L. Wall, J. J. Bollinger, and A. M. Rey, Nat. Phys. 13, 781 (2017).

[19] E. J. Meier, J. Ang'ong'a, F. A. An, and B. Gadway, arXiv:1705.06714.

[20] S. Pancharatnam, Proc. Indian Acad. Sci. A 44, 247 (1956).

[21] V. Bargmann, J. Math. Phys. (N.Y.) 5, 862 (1964).

[22] M. V. Berry, Proc. R. Soc. A 392, 45 (1984).

[23] Y. Aharonov and J. Anandan, Phys. Rev. Lett. 58, 1593 (1987).

[24] N. Mukunda and R. Simon, Ann. Phys. (N.Y.) 228, 205 (1993).

[25] P. D. Lax, Linear Algebra and its Applications, 2nd Ed. (Wiley, New Jersey, 2007), Chap. 10.

[26] H. P. Robertson, Phys. Rev. 46, 794 (1934).

[27] J.-M. Levy-Leblond, Ann. Phys. (N.Y.) 101, 319 (1976).

[28] See Supplemental Material at http://link.aps.org/ supplemental/10.1103/PhysRevLett.120.230402 for detailed properties of the unitary and overlap uncertainty relations.

[29] I. Todhunter, Spherical Trigonometry, 5th Ed. (Macmillan, London, 1886), http://www.gutenberg.org/ebooks/19770.

[30] S. M. Barnett and S. Croke, Adv. Opt. Photonics 1, 238 (2009).

[31] M. M. Wilde, Quantum Information Theory, 2nd Ed. (Cambridge University Press, Cambridge, 2017), Chap. 9.

[32] R. B. Patel, J. Ho, F. Ferreyrol, T. C. Ralph, and G. J. Pryde, Sci. Adv. 2, e1501531 (2016).

[33] H. Buhrman, R. Cleve, J. Watrous, and R. de Wolf, Phys. Rev. Lett. 87, 167902 (2001).

[34] J. C. Loredo, O. Ortíz, R. Weingärtner, and F. De Zela, Phys. Rev. A 80, 012113 (2009).

[35] L. Xiao, K. Wang, X. Zhan, Z. Bian, J. Li, Y. Zhang, P. Xue, and A. K. Pati, Opt. Express 25, 17904 (2017). 